\title{
Morphological and Kinematical Modelling of PNe and Nova Shells Using XSHAPE
}

\author{
D. J. Harman, M. Bryce, T. J. O'Brien, J. Meaburn \\ Jodrell Bank Observatory, Department of Physics and Astronomy, \\ University of Manchester, Macclesfield, Cheshire, SK11 9DL
}

\begin{abstract}
XSHAPE has been developed to aid the better understanding of the 3D morphologies and kinematics of PNe and novae, by producing models which closely predict the observed 2D images and long-slit spectra of these objects. Early successes include plausible simulations for the elliptical PN Sa 2-21 and the nova HR Del.
\end{abstract}

\section{Introduction}

XSHAPE has been developed (from the original SHAPE code by Wolfgang Steffen) primarily to model long slit spectra and images obtained with the Manchester echelle spectrometer (MES; Meaburn et al. 1984). A number of geometrical shapes, including spheres, ellipsoids and closed and open-ended hour-glasses etc, can be used individually or combined to model, in $3 \mathrm{D}$, basic or complex structures including rings and polar axis density gradients.

A geometrical shape (or shapes) such as an ellipsoid can be rotated to match the position angle (PA) and inclination of the nebula that is being modeled. Parameters such as the major and minor axis lengths, FWHM of line profiles from the shell, polar axis emission gradients and the nebular expansion velocity (based on $v \propto r$ ) can be altered until a match with observations is achieved. A simulation of the spectrometer slit can be placed across the model at any position and PA to produce a position velocity (pv) array to match the observed long slit spectra.

Additional features can be included to increase realism such as seeing conditions, instrumental velocity resolution, filter bandpass and random clumpiness. The whole application is held together in a graphical user interface and the predicted 2D images and pv spectra can be generated in a variety of file formats (Harman 2001).

\section{Planetary Nebulae - Sa 2-21}

The morphology and kinematics of Sa 2-21, a hitherto little studied ellipsoidal $\mathrm{PN}$, have been determined by comparing XSHAPE predictions with high resolution images and long slit MES spectra from the Anglo Australian Telescope (Harman, Bryce \& Holloway 2001), see Fig 1. The structure of Sa 2-21 has been modeled to resemble what is observed in both the [OIII] $\lambda 5007 \AA$ and [NII] $\lambda 6584 \AA$ emission lines. The [OIII] structure consists of a dense equatorial re- 


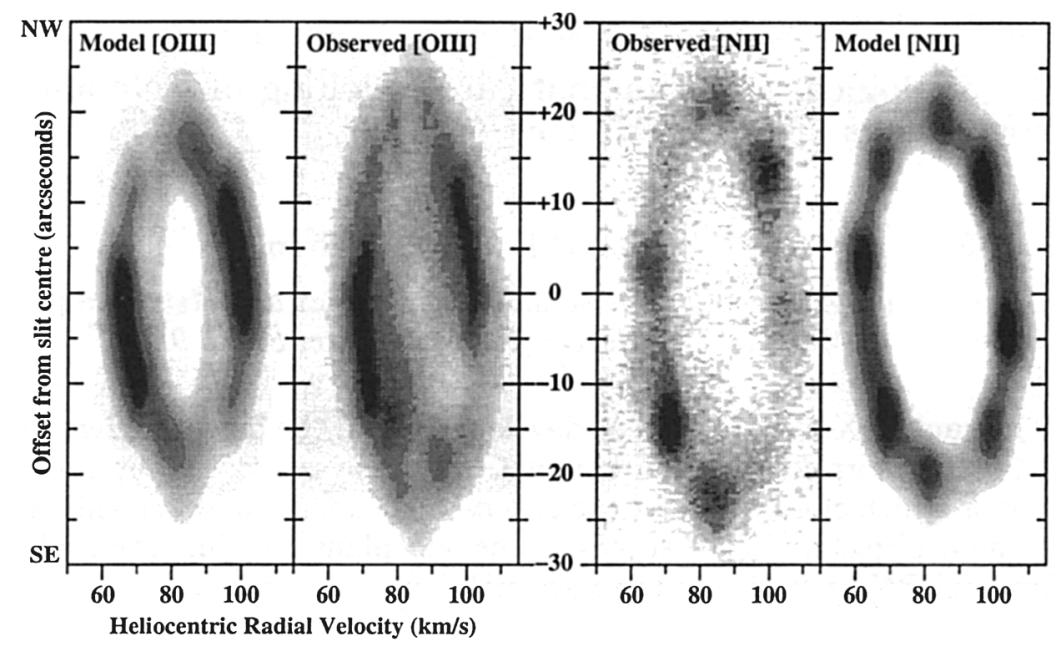

Figure 1. Model long slit spectra with corresponding observed [OIII] and $[\mathrm{NII}]$ data in the inner two panels. Slit position is at $\mathrm{PA}=125^{\circ}$ through the centre of Sa 2-21.

gion, less dense tropical regions and very low density poles. A faint halo in [OIII] is also seen. The [NII] emission is concentrated into rings coincident with the changes in density in the [OIII] structure.

\section{Nova Shells - HR Del}

XSHAPE is being used to investigate the similarities between nova shells and those of PNe. HR Del (Nova Del 1967) was investigated using a combination of HST imaging in $\mathrm{H} \alpha$, [NII] $\lambda 6584 \AA$ and [OIII] $\lambda 5007 \AA$, and WHT/ISIS long slit spectra. XSHAPE allowed us to develop a model that produces high resolution images comparable with HST data and spectra that when convolved with appropriate seeing conditions and velocity resolution compare favorably with observations, (Harman, O'Brien \& Meaburn 2001). The basic model is that of a closed hour glass (bi-lobal shape) with an equatorial ring and polar rings. The [OIII] model has emission concentrated into the polar rings, whereas in the $\mathrm{H} \alpha$ model, the emission is more evenly spread throughout the main shell and equatorial ring.

\section{References}

Meaburn, J., Blundell, B., Carling, R., Gregory, D. F., Keir, D., \& Wynne, C. G. 1984, MNRAS, 210, 463

Harman, D. J. 2001, PhD thesis, Univ. Manchester

Harman, D. J., Bryce, M., \& Holloway, A. J. 2001, MNRAS, in prep

Harman, D. J., O'Brien, T. J., \& Meaburn, J. 2001, MNRAS, in prep 